\title{
Effect of Deep Eutectic Solvent Nanostructure on Phospholipid Bi-
}

\section{layer Phases}

\author{
Saffron J. Bryant, ${ }^{1}$ Rob Atkin, ${ }^{2}$ and Gregory G. Warr ${ }^{1 *}$ \\ 1. School of Chemistry and Australian Institute for Nanoscale Science and Technology, The University of Sydney, \\ NSW 2006, Australia \\ 2. School of Molecular Sciences, The University of Western Australia, WA 6009, Australia
}

\begin{abstract}
Phospholipids are shown by solvent penetration experiments to form lamellar phases and spontaneously spawn vesicles in a wide range of deep eutectic solvents (DESs) composed of alkylammonium halide salts and glycerol or ethylene glycol, which are shown to be nanostructured by X-ray scattering. In contrast with molecular solvents, the chain melting temperature of each phospholipid, which determines the stability of the swellable bilayer phase, depends on the structure of the cation, anion and molecular H-bond donor that constitute the DES. Chain melting is most sensitive to the length of the alkyl chain of the cation, which is partitioned between apolar domains in the bulk, nanostructured DES and those within the lipid bilayer. This is moderated by the structures of the anion and the molecular hydrogen bond donor, which determine the extent of polar/apolar segregation in the bulk liquid.
\end{abstract}

* Corresponding Author: Tel.: +61 029351 2106. E-mail: gregory.warr@ sydney.edu.au

\section{Introduction}


Cell membranes are vital to life. ${ }^{1-4}$ They are composed mainly of a phospholipid bilayer, which separates and protects the cell interior from the external environment. ${ }^{5}$ Vesicles, which are formed from a bilayer membrane encapsulating solvent, have similar structures. They make excellent models for cell-like compartmentalization, ${ }^{5}$ can be effective as nanocapsules, ${ }^{6}$ as isolated nanoreactors ${ }^{4,7}$ and can be used for drug delivery. ${ }^{8}$

Phospholipid assembly in water ${ }^{9}$ and other polar solvents ${ }^{10-11}$ occurs due to the solvophobic effect. ${ }^{9,12}$ The two-tailed structure and packing geometry of phospholipids leads to their assembly into locally planar structures,${ }^{9}$ and phase diagrams are dominated by bilayer phases, such as lamellar, bicontinuous cubic phases, and vesicles. ${ }^{13-16}$ In water, phospholipids undergo a "chain melting" phase transition upon warming from the highly ordered $L_{\beta}$ phase (in which the alkyl chains within the bilayer are crystalline) into an $\mathrm{L}_{\alpha}$ phase where the chains have liquid-like organisation. ${ }^{17-18}$ The resultant flexibility of the $\mathrm{L}_{\alpha}$ phase allows solvent penetration and swelling, ultimately leading to vesicle formation. The transition temperature in water depends on the length and saturation of the lipid alkyl tails, with longer tails and increased saturation leading to higher transition temperatures. ${ }^{13}$

Phospholipid assembly into the $\mathrm{L}_{\alpha}$ phase has been studied in various non-aqueous but polar, $\mathrm{H}$ bonding molecular solvents. ${ }^{19}$ The chain-melting temperature of 1,2-dipalmitoyl-sn-glycero-3phosphocholine (DPPC) has been found to be $41^{\circ} \mathrm{C}$, regardless of whether the solvent was glycerol, ethylene glycol or water. ${ }^{19}$ Lamellar phase formation by egg phosphatidylcholine in formamide and 1,3-propanediol has also been observed ${ }^{11}$ but chain melting temperatures were not reported. Recent work has demonstrated that that bilayer assembly is not limited to molecular solvents but can also occur in ionic liquids by both lipids ${ }^{10,20}$ and synthetic amphiphiles. ${ }^{21}$

Deep eutectic solvents (DESs) are composed of a mixtures of salts, or of salts and H-bond donors, which form a eutectic when mixed together at a well-defined stoichiometry. Melting points are sometimes hundreds of degrees lower than those of the individual components. ${ }^{22}$ DESs are interesting solvents because they have low-toxicity, are cheap, and are easy to prepare. ${ }^{23-25} \mathrm{We}$ have recent- 
ly shown that phospholipid vesicles can be formed in the most widely studied DES, ${ }^{22,26} 1: 2$ mol:mol choline chloride-urea. ${ }^{27}$ Solvophobic self-assembly of surfactants into micelles and other aggregates in this, and other DESs has also been reported. ${ }^{28-30}$ Control over the properties of DESs is achieved by changing the structure of the ions (just as is done with ionic liquids) as well as the H-bond donor, e.g., from choline to ethylammonium chloride, or urea to acetamide ${ }^{31}$ or glycerol. ${ }^{24}$ We have recently reported the properties and structures of a class of DESs formed from mixtures of alkylammonium bromide salts with glycerol ${ }^{32}$ and urea. $^{33}$ Unlike choline-based DESs, the cations in these systems are amphiphilic, which may lead to liquid nanostructure comprised of polar and non-polar domains such as that seen in alkylammonium-based ionic liquids. ${ }^{34-36}$ There is strong evidence for the persistence of such amphiphilic nanostructure when alkylammonium ionic liquids are diluted with water, ${ }^{37}$ glycerol, ${ }^{38}$ or aliphatic alcohols,${ }^{39}$ or are mixed with alkylammonium chloride salts. ${ }^{40}$ Alkylammonium bromide-based DESs also show layering at interfaces that has previously been correlated with bulk amphiphilic nanostructure. ${ }^{32}$

Like polar, H-bonding molecular solvents, ionic liquids such as ethylammonium nitrate support the formation of lipid bilayer phases. ${ }^{41}$ Nanostructure in ionic liquids strongly affects the ability of phospholipids to form stable bilayer phases and vesicle dispersions. ${ }^{20}$ Such non-aqueous solvents provide a potential means of stabilizing bilayers in extreme environments such as for cryopreservation, as examined by Choi et al. ${ }^{42}$ and for understanding survival under desiccation. ${ }^{43}$

This work examines the effects of DES cation chain length, anion, and hydrogen bond donor type, on the stability and formation of phospholipid lamellar phases, and provides insight into the factors that stabilize fluid lipid bilayer phases and dispersions. 


\section{Experimental Section}

Deep eutectic solvents were prepared by separately drying the components using rotary evaporation and freeze drying and then mixing them at a 1:2, salt:H-bond donor ratio. The mixtures were heated to $70{ }^{\circ} \mathrm{C}$ to speed dissolution of the salts and then stored under dry conditions. Water content was maintained below $0.1 \mathrm{wt} \%$, as measured by Karl Fischer titration.

Small and wide-angle X-ray scattering was carried out on an Anton-Paar SAXSess in point collimation using $1 \mathrm{~mm}$ quartz capillary cells. All scattering patterns were recorded at $25^{\circ} \mathrm{C}$.

Figure 1 shows the molecular and salt components of the DESs used, which include ethylene glycol (Sigma Aldrich, 99\%), and glycerol (Sigma Aldrich, 99\%) as hydrogen bond donors. The ethylammonium, propylammonium, and butylammonium salts were synthesised by adding either hydrochloric (Ajax, 32\%) or hydrobromic (Ajax, 48\%) acid dropwise to equimolar amounts of ethylamine (Sigma Aldrich, 70\%), propylamine (Sigma Aldrich, 98\%), butylamine (Merck, 99\%), or pentylamine (Fluka, 98\%) and then removing excess water by rotary evaporation and freeze drying.

Four different phosphatidylcholine lipids were used, as shown in Figure 1. Three contained saturated alkyl chains of differing lengths; 1,2-dimyristoyl-sn-glycero-3-phosphocholine (DMPC: $\mathrm{C}_{14}$ ), 1,2-dipalmitoyl-sn-glycero-3-phosphocholine （DPPC: $\quad \mathrm{C}_{16}$ ), 1,2-distearoyl-sn-glycero-3phosphocholine (DSPC: $\mathrm{C}_{18}$ ), while the fourth, egg PC, is a mixture containing 1-palmitoyl-2oleoyl-sn-glycero-3-phosphocholine (POPC) as its majority constituent. All lipids were purchased from Sigma-Aldrich with $\geq 99 \%$ purity, and were used as received.

Isothermal solvent penetration experiments were carried out as previously described ${ }^{27,44}$ to examine lyotropic phase formation over a wide range of concentrations. Briefly, a small amount of lipid was placed between a microscope slide and a coverslip to produce a film approximately $40 \mu \mathrm{m}$ thick. A drop of each solvent (DES or hydrogen bond donor) was placed on the outer edge of the coverslip and drawn into contact with the lipid by capillary action to create a concentration gradient from pure 
DES to pure lipid. Polarising optical microscopy was used to identify phases formed across the concentration gradient based on well-known optical signatures for each phase. ${ }^{45}$ The microscope stage was heated from room temperature to $353 \mathrm{~K}$ to examine phase changes with temperature. The temperature stage is accurate to $\pm 0.1{ }^{\circ} \mathrm{C}$ and previous work has demonstrated that polarizing optical microscopy is a valid method of determining transition temperatures that are consistent with those found via differential scanning calorimetry. ${ }^{46-47}$

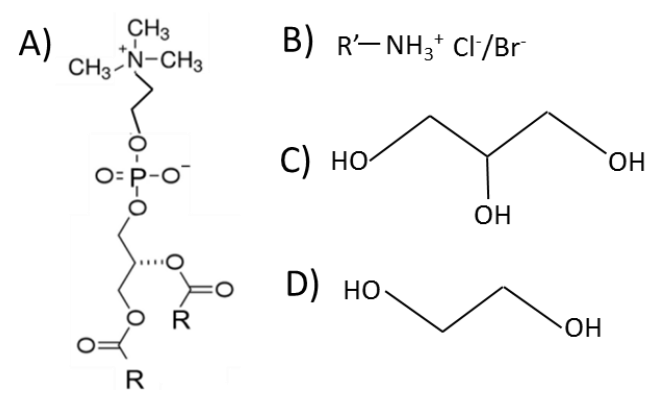

Figure 1. Structures of the phospholipids (A), salts (B), and H-bond donors glycerol (C) and ethylene glycol (D) used in this work, where $\mathrm{R}=\mathrm{C}_{13} \mathrm{H}_{27}$ (DMPC); $\mathrm{C}_{15} \mathrm{H}_{31}$ (DPPC); $\mathrm{C}_{17} \mathrm{H}_{35}$ (DSPC); or a mixture (egg PC), and $\mathrm{R}^{\prime}=\mathrm{C}_{2} \mathrm{H}_{5}(\mathrm{EA}), \mathrm{C}_{3} \mathrm{H}_{7}(\mathrm{PA}), \mathrm{C}_{4} \mathrm{H}_{9}(\mathrm{BA})$, or $\mathrm{C}_{5} \mathrm{H}_{11}(\mathrm{PeA})$. 


\section{Results and Discussion}

Egg PC forms swollen lamellar $\left(\mathrm{L}_{\alpha}\right)$ phases in all of the molecular H-bond donors and DESs examined. This assignment is made on the basis of myelinic figures and maltese crosses, which are a signature texture of lamellar swelling and vesicle formation. ${ }^{44,48}$ Representative results are shown in

Figure 2. Heating above room temperature further swelled the egg PC $\mathrm{L}_{\alpha}$ phases.
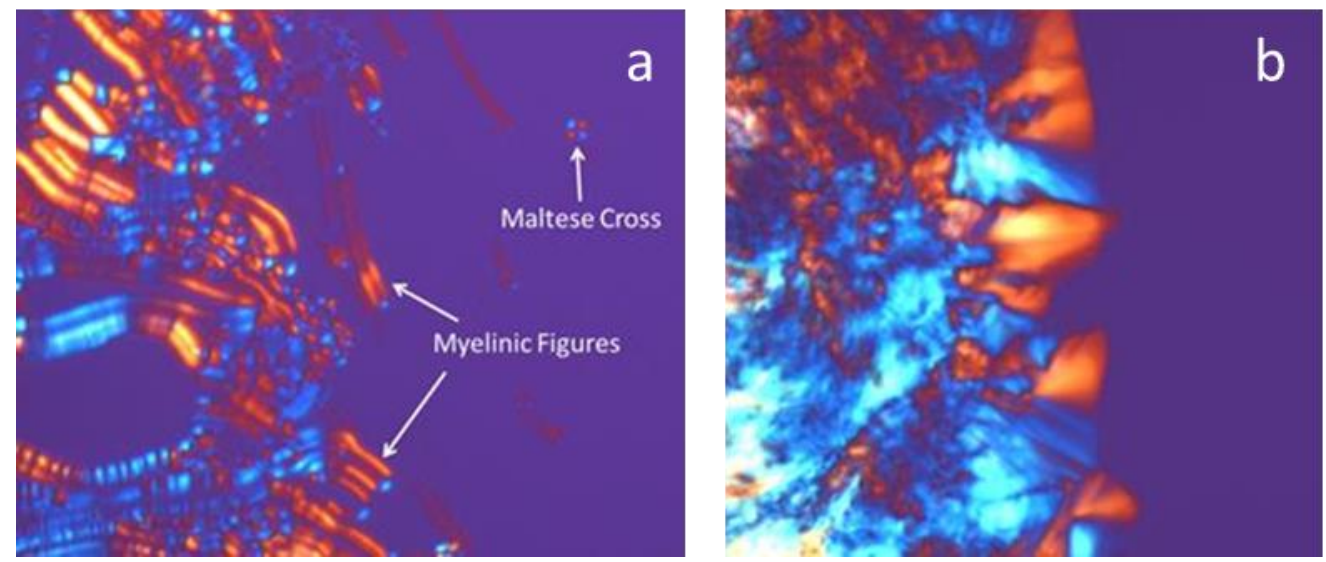

Figure 2. Representative isothermal penetration scans of (a) Egg PC in BABr-Gly at $23{ }^{\circ} \mathrm{C}$ showing a swollen lamellar phase, arrows indicate myelinic figures and a maltese cross (vesicle), and (b) DMPC at $34{ }^{\circ} \mathrm{C}$ in ethylene glycol, showing hexagonal phase texture.

In contrast, all the saturated-chain lipids required heating in order to form a swellable lamellar $\left(\mathrm{L}_{\alpha}\right)$ phase and spawn vesicles. This minimum solvent penetration temperature is commonly equated to the chain melting temperature of the lipids, $\mathrm{T}_{\mathrm{m}}$. In almost all cases this transition was from crystals directly to a swollen $\mathrm{L}_{\alpha}$ phase, except for DMPC in ethylene glycol, which instead formed a hexagonal phase (see Figure 2(b)), and which did not change with further heating. No hexagonal phase was observed for any of the other lipid/solvent combinations. In PABr-glycerol, both DMPC and DPPC first formed an unswollen $\mathrm{L}_{\beta}$ (lamellar gel) phase, before swelling and budding vesicles at $\mathrm{T}_{\mathrm{m}}$. Chain melting temperatures for all saturated-chain lipids in molecular and deep eutectic solvents are listed in Table 1. 
Table 1. Transition temperatures $\left({ }^{\circ} \mathrm{C}\right)$ of lipids in DESs and H-bond donors into a swellable phase from isothermal penetration scans, with literature comparisons where available. Also shown are previous observations for comparable alkylammonium ionic liquids. The high-temperature phase is lamellar $\left(\mathrm{L}_{\alpha}\right)$, unless otherwise noted: hex. $=$ hexagonal and unk. $=$ unknown. ${ }^{20}$ (Behavior below $23^{\circ} \mathrm{C}$ was not examined).

\begin{tabular}{l|cccc}
\hline & $\boldsymbol{E} \boldsymbol{g} \boldsymbol{g} \boldsymbol{P C}$ & $\boldsymbol{D M P C}$ & $\boldsymbol{D P P C}$ & $\boldsymbol{D S P C}$ \\
\hline Water & $<23$ & $23\left(23^{13}\right)$ & $41\left(41^{13}\right)$ & $56\left(54^{13}\right)$ \\
EG & $<23$ & $24($ hex. $)$ & $42\left(41^{19}\right)$ & 55 \\
Glycerol & $<23$ & $23\left(23^{49}\right)$ & $42\left(41^{19}\right)$ & $55\left(56^{49}\right)$ \\
EACl-EG & $<23$ & 35 & 43 & 57 \\
EACl-Gly & $<23$ & 28 & 43 & 56 \\
EABr-EG & $<23$ & 45 & 55 & 57 \\
EABr-Gly & $<23$ & 50 & 55 & 56 \\
EAN ${ }^{20}$ & $<23$ & $49($ unk.) & $50($ hex.) & 72 \\
EAF & $<23$ & 30 & 50 & 66 \\
PABr-EG & $<23$ & 54 & 62 & 70 \\
PABr-Gly & $<23$ & $48^{*}$ & $75^{*}$ & 57 \\
PAN ${ }^{20}$ & $<23$ & $45($ unk.) & $50($ hex.) & 72 \\
BABr-EG & $<23$ & 35 & 44 & 54 \\
BABr-Gly & $<23$ & 26 & 40 & 54 \\
PeABr-EG & $<23$ & $<23$ & 35 & 48 \\
PeABr-Gly & $<23$ & $<23$ & 38 & 49 \\
\hline * DMPC melts at $42{ }^{\circ} \mathrm{C}$ and DPPC at $43^{\circ} \mathrm{C}$ into an L ${ }_{\beta}$ or gel phase.
\end{tabular}

As in water, $\mathrm{T}_{\mathrm{m}}$ in both H-bond donor solvents and DESs increases with saturated alkyl chain length. ${ }^{13}$ Although replacing water with glycerol or ethylene glycol had no effect on $\mathrm{T}_{\mathrm{m}},{ }^{19} \mathrm{DESs}$ were found to increase $\mathrm{T}_{\mathrm{m}}$ markedly, by up to $20^{\circ} \mathrm{C}$ in some cases. As noted in Table 1, DMPC and DPPC in PABr-Gly both first formed an $\mathrm{L}_{\beta}$ phase below the chain-melting transition to a swellable $\mathrm{L}_{\alpha}$ phase. (Optical micrographs of penetration scans of these phases at three temperatures are provided as electronic supplementary information.)

Figure 3 shows the effect of DES cation alkyl chain length on $T_{m}$ of saturated lipids for DESs formed using either ethylene glycol and glycerol H-bond donors. For both H-bond donors, $\mathrm{T}_{\mathrm{m}}$ first increases with ethylammonium and propylammonium cations, and then decreases upon further in- 
creasing cation alkyl chain length. The increase is larger with bromide than chloride salts. The effect of changing the DES is most pronounced in DMPC, and generally diminishes with increasing saturated lipid acyl chain length.
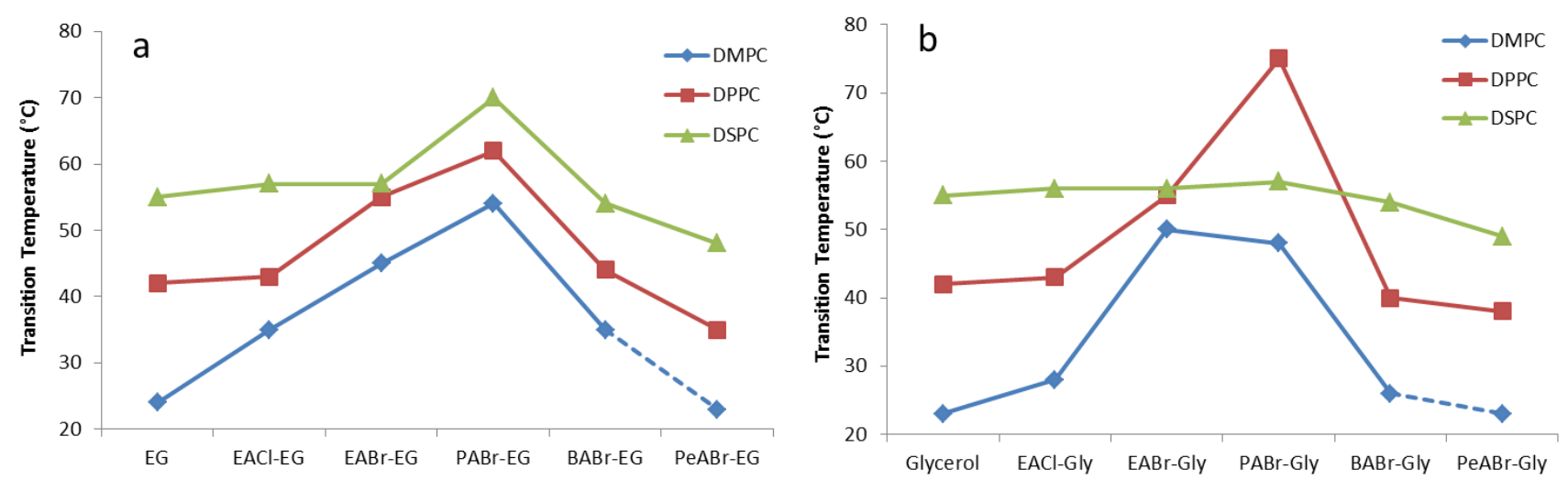

Figure 3. Minimum swelling temperatures ( $\mathrm{L}_{\alpha}$ formation) of the saturated lipids in DESs using (a) ethylene glycol and (b) glycerol as H-bond donors.

The increase in $T_{m}$ in DESs compared to water, ethylene glycol and glycerol implicates the cations and anions in stabilizing the ordered acyl chain state over L $\alpha$. The high ionic strength of DESs (and ionic liquids) is expected to screen repulsions between adjacent lipid headgroups, thereby reducing the driving force for $\mathrm{L}_{\alpha}$ phase formation and the associated increase in head-group area, ${ }^{50-52}$ but the observed effects are also ion specific. This is attributed to a combination of the alkylammonium cations acting as $\mathrm{H}$-bond donors to the phosphate and carbonyl oxygens of the lipids, similar to the role of water in aqueous systems, ${ }^{53-54}$ and to anion association with the quaternary ammonium charged group of the lipid. This interpretation is consistent with the reduced effect of solvent on $\mathrm{T}_{\mathrm{m}}$ as lipid acyl chain length is increased. As $\mathrm{T}_{\mathrm{m}}=\Delta H / \Delta S$ is the coexistence temperature of the unswollen and swollen phases (i.e. $\Delta G=0$ ), the contribution of tails to both $\Delta H$ and $\Delta S$ increases with chain length, accounting for the reduced sensitivity to changes in the head group region, where solvent effects operate. 
Effect of Anion. The chain melting transition occurs at higher temperatures in bromidecontaining than chloride-containing ethylammonium DESs. This is consistent with the well-known stronger binding of bromide ions to quaternary ammonium head groups in surfactant micelles, which are analogous to the choline charged centre of these lipids. The anions from our previous work on ionic liquids (see Table 1) also fit into this trend ${ }^{20}$ further supporting the essential role of the ionic components of the DESs. In ILs, comparably strongly-binding nitrate and bromide have the highest $\mathrm{T}_{\mathrm{m}}$ values, and the weakest associating anion, formate, has the lowest. ${ }^{55-56}$ While the majority of studies in aqueous systems have focused on the effect of the cation, recent investigations have found that the effect of anions tends to follow the Hofmeister series. ${ }^{57-59}$

Effect of Cation. Both electrostatic and H-bonding considerations favour strong solvation of the phosphate moieties by the cation ammonium groups rather than ethylene glycol and glycerol. The complex cation alkyl chain length dependence observed for $\mathrm{T}_{\mathrm{m}}$ also suggests a lesser role for the $\mathrm{H}$ bond donor molecules.

The origin of this behavior may be understood by considering the amphiphilic nanostructure of alkylammonium-based DESs. Figure 4 shows the X-ray scattering patterns of the pure DESs with both glycerol and ethylene glycol. These DESs all exhibit a low-angle (low $Q$ ) scattering peak that begins as a shoulder for ethylammonium, but becomes more pronounced and shifts to lower $Q$ with increasing cation alkyl chain length. This feature has been identified with the development of amphiphilic nanostructure alkylammonium ionic liquids ${ }^{34,60-63}$ and solutions, ${ }^{36,38,40}$ arising from the segregation of non-polar alkyl chains from the electrostatic and H-bonding network of the ammonium and anion charged centres. 

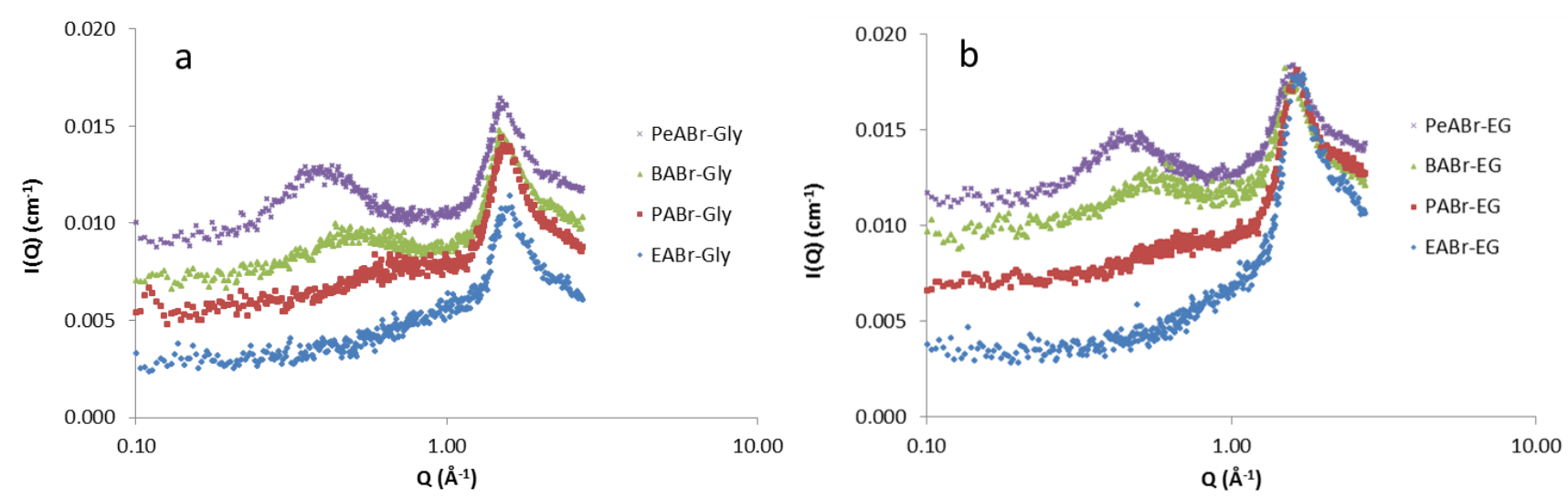

Figure 4. X-ray scattering patterns of alkylammonium bromide deep eutectic solvents with (a) glycerol and (b) ethylene glycol H-bond donors. (Data have been offset on the y-axis for clarity).

These results are strikingly similar to those of Greaves et $a l .{ }^{63}$ for primary alkylammonium nitrate and formate ionic liquids. The sizes of the nanostructure in these DESs and comparable ionic liquids, calculated from the peak position from X-ray or neutron scattering using $D^{*}=2 \pi / Q$, are given in Table 2. In both cases $D^{*}$ increase monotonically with cation chain length as expected for nonpolar domains formed by alkyl chain association.

Table 2. Nanostructure size ( $($ ) of alkylammonium bromide deep eutectic solvents based on the primary peak position, $D^{*}$, compared with those found in corresponding alkylammonium protic ionic liquids. (The primary peak position for the ethylammonium bromide DESs was not distinct enough to identify.)

\begin{tabular}{c|cccc}
\hline & Glycerol & Ethylene Glycol & $\begin{array}{c}\text { Nitrate ionic liq- } \\
\text { uids }^{\mathbf{6}^{3}}\end{array}$ & $\begin{array}{c}\text { Formate ionic }^{6} \\
\text { liquids }^{63}\end{array}$ \\
\hline EABr & - & - & $9.0\left(9.7^{64}\right)$ & 8.4 \\
PABr & 9.1 & 9.1 & $11.7\left(11.6^{64}\right)$ & - \\
BABr & 11.7 & 11 & 14.0 & 13.2 \\
PeABr & 16 & 14 & 15.9 & 15.2 \\
\hline
\end{tabular}

For a given anion (and in DESs, H-bond donor), the strength of the solvophobic driving force and the extent of nanosegregation into polar and non-polar domains increases with increasing cation alkyl chain length, just as it does in protic ionic liquids. In other words, solute species like the phosphocholine head group are less able to recruit solvent species for solvation and swelling as chain length increases, as this would disrupt the existing liquid nanostructure. Hence $\mathrm{T}_{\mathrm{m}}$ increases. 
With even longer alkyl chains, the cation begins to act as a cosurfactant for the lipid. Its ammonium group may remain H-bonded to the phosphate or carbonyl moiety while its alkyl group mixes into the hydrophobic core of the lipid bilayer. This effect is well known in aqueous systems where, for example, increasing the chain length of carboxylate counterions increases their binding to quaternary ammonium cationic surfactant films. ${ }^{56}$ Similarly, aliphatic alcohols act as cosurfactants in aqueous micelles above a threshold chain length of 3-4, ${ }^{65-66}$ and alcohol partitioning into lipid bilayers increases with chain length. ${ }^{67-68}$ In this work, cation alkyl chain penetration into lipid chains in the bilayer stabilizes the fluid $\mathrm{L}_{\alpha}$ state by disrupting crystalline packing of lipid alkyl chains, which reduces $\mathrm{T}_{\mathrm{m}}$.

Effect of H-Bond Donor. Although not directly involved in solvating the lipid headgroups, Hbond donors are critical to stabilizing the liquid state and the bulk structure of DESs. Glycerol-based DESs are expected to exhibit stronger solvophobic effects than their ethylene glycol counterparts on several grounds. First is the Gordon parameter, which is a good indicator of an ionic or molecular solvent's capacity to support solvophobic self assembly; ${ }^{69}$ Glycerol has a larger Gordon parameter than ethylene glycol. ${ }^{70}$ Second, glycerol has more hydrogen bonding sites than ethylene glycol, making it less tolerant of hydrocarbon chains. This expectation is also supported by the empirical observation that critical micelle concentrations of surfactants in glycerol are often less than half those in ethylene glycol. ${ }^{71-74}$

That DMPC forms a hexagonal phase in ethylene glycol, whilst in water and glycerol it forms a swellable lamellar phase is also indicative of a weaker solvophobic effect. A hexagonal phase reveals the packing parameter ${ }^{75}$ of DMPC in ethylene glycol is smaller than in either water or glycerol, consistent with higher hydrocarbon solubility, or at least a greater area of hydrocarbon/solvent contact. 
Unlike the situation with H-bond donors alone, in these DESs there are no clear, systematic differences in behaviour between glycerol and ethylene glycol. Previous studies of water in ethylammonium nitrate ${ }^{37}$ and glycerol in ethylammonium formate ${ }^{38}$ elucidated similar liquid nanostructures. The polar and non-polar domains present in the pure ionic liquids is retained, with water or glycerol solubilized by the polar domains. Ethylene glycol is expected to behave likewise.

This suggests that these DESs mirror the behaviour we have found previously for protic ionic liquids; the solvophobic effect and liquid nanostructure are dominated by electrostatics, and the H-bond network structure is a secondary scaffold - provided in these DESs by the molecular H-bond donor that accommodates the preferred arrangement of charges. ${ }^{76}$ The direct influence of the H-bond donor on lipid behavior is thus attenuated within a DES.

\section{Summary and Conclusions}

Phospholipids form a swellable lamellar phase and spontaneously spawn vesicles in a range of deep eutectic solvents. The minimum temperature for fluid lamellar phase formation is dependent on both the cation and anion type in the DES but, for the samples investigated here, depends less strongly on the H-bond donor.

The stability of the $\mathrm{L}_{\alpha}$ phase is particularly sensitive to the hydrocarbon chain length of the alkylammonium DES cation. The minimum swelling temperature for formation $\mathrm{L}_{\alpha}$ phases first increases and then decreases with increasing cation chain length. This is a result of a competition between the nonpolar domains (solvophobic nanostructure) in the DES bulk and those in the lipid bilayer. For sufficiently long chains, the cation acts as a cosurfactant for the lipid, stabilizing $\mathrm{L}_{\alpha}$ at lower $\mathrm{T}_{\mathrm{m}}$. This suggests that a more direct investigation of DES nanostructure is warranted, and this is currently in progress.

The virtual ubiquity of a swellable lamellar phase and vesicles in these DESs contrasts with our recent observations on protic ionic liquids. There, phospholipid vesicle stability was found to be 
highly dependent on cation amphiphilicity, and liquid nanostructure was an undesirable feature of the solvent. ${ }^{20}$ This suggests that DESs may be advantageous as solvents for complex solutes and amphiphiles, possibly as a consequence of the separation of electrostatic and H-bonding duties between anion and molecular components.

Only a very limited range of molecular solvents have ever been shown to exhibit a solvophobic effect and support amphiphile self-assembly, although studies of ionic liquids have expanded that family substantially. ${ }^{9-10,13,21,77}$ This work significantly broadens the set of available solvents that can drive solvophobic self-assembly, and reveals new control mechanisms for stabilizing bilayer structures in nonaqueous solution. Using DESs, lipids and synthetic amphiphiles may be used to create nanocapsules and nanoreactors to control chemical processes and substrate release or delivery. ${ }^{78}$ These results broaden the potential range of extreme environments under which bilayer membranes can exist, including for cryopreservation ${ }^{42}$ or under extreme desiccation, ${ }^{43}$ the existence of extremophiles, ${ }^{79}$ including those that might evolve on planets other than Earth. ${ }^{80-81}$

\section{Acknowledgements}

This work was supported by a Discovery Project grant from the Australian Research Council. SJB thanks Australian Institute of Nuclear Science and Engineering for a postgraduate research award.

Supporting Information: Additional polarizing optical microscopy images and X-ray scattering curves.

\section{References}

1. Bitbol, M.; Luisi, P. L., Autopoiesis with or without Cognition: Defining Life at Its Edge. J. $R$. Soc., Interface 2004, 1 (1), 99-107.

2. Black, R. A.; Blosser, M. C.; Stottrup, B. L.; Tavakley, R.; Deamer, D. W.; Keller, S. L., Nucleobases Bind to and Stabilize Aggregates of a Prebiotic Amphiphile, Providing a Viable Mechanism for the Emergence of Protocells. Proc. Natl. Acad. Sci. U.S.A. 2013, 110 (33), 1327213276.

3. Carrara, P.; Stano, P.; Luisi, P. L., Giant Vesicles "Colonies": A Model for Primitive Cell Communities. ChemBioChem 2012, 13 (10), 1497-1502. 
4. Chen, I. A.; Walde, P., From Self-Assembled Vesicles to Protocells. Cold Spring Harbor Perspect. Biol. 2010, 2 (7), a002170.

5. Cornell, B. A.; Fletcher, G. C.; Middlehurst, J.; Separovic, F., The Lower Limit to the Size of Small Sonicated Phospholipid Vesicles. Biochim. Biophys. Acta, Biomembr. 1982, 690 (1), 15-19.

6. Ruysschaert, T.; Germain, M.; Gomes, J.; Fournier, D.; Sukhorukov, G. B.; Meier, W.; Winterhalter, M., Liposome-Based Nanocapsules. Ieee Transactions on Nanobioscience 2004, 3 (1), 49-55.

7. Walde, P.; Ichikawa, S., Enzymes inside Lipid Vesicles: Preparation, Reactivity and Applications. Biomol. Eng. 2001, 18 (4), 143-177.

8. Jain, S.; Jain, V.; Mahajan, S. C., Lipid Based Vesicular Drug Delivery Systems. Advances in Pharmaceutics 2014, 2014, 12.

9. Tanford, C., The Hydrophobic Effect and the Organization of Living Matter. Science 1978, 200 (4345), 1012-1018.

10. Gayet, F.; Marty, J.-D.; Brûlet, A.; Viguerie, N. L.-d., Vesicles in Ionic Liquids. Langmuir 2011, 27 (16), 9706-9710.

11. McIntosh, T. J.; Magid, A. D.; Simon, S. A., Range of the Solvation Pressure between Lipid Membranes: Dependence on the Packing Density of Solvent Molecules. Biochemistry 1989, 28 (19), 7904-7912.

12. Hanahan, D. J., A Guide to Phospholipid Chemistry. Oxford University Press, USA: 1997.

13. Koynova, R.; Caffrey, M., Phases and Phase Transitions of the Phosphatidylcholines. Biochim. Biophys. Acta, Rev. Biomembr. 1998, 1376 (1), 91-145.

14. Stano, P.; Luisi, P. L., Achievements and Open Questions in the Self-Reproduction of Vesicles and Synthetic Minimal Cells. Chem. Commun. 2010, 46 (21), 3639-3653.

15. Woodka, A. C.; Butler, P. D.; Porcar, L.; Farago, B.; Nagao, M., Lipid Bilayers and Membrane Dynamics: Insight into Thickness Fluctuations. Phys. Rev. Lett. 2012, 109 (5), 058102.

16. Ravoo, B. J.; Weringa, W. D.; Engberts, J. B. F. N., Design and Characterization of Synthetic Bilayer Vesicles with a Polymerized Inner Bilayer Leaflet. Langmuir 1996, 12 (24), 5773-5780.

17. Grabielle-Madelmont, C.; Perron, R., Calorimetric Studies on Phospholipid-Water Systems: I. Dl-Dipalmitoylphosphatidylcholine (Dppc)—Water System. J. Colloid Interface Sci. 1983, 95 (2), 471-482.

18. Tardieu, A.; Luzzati, V.; Reman, F. C., Structure and Polymorphism of the Hydrocarbon Chains of Lipids: A Study of Lecithin-Water Phases. J. Mol. Biol. 1973, 75 (4), 711-733.

19. McDaniel, R. V.; McIntosh, T. J.; Simon, S. A., Nonelectrolyte Substitution for Water in Phosphatidylcholine Bilayers. Biochim. Biophys. Acta, Biomembr. 1983, 731 (1), 97-108.

20. Bryant, S. J.; Wood, K.; Atkin, R.; Warr, G. G., Effect of Protic Ionic Liquid Nanostructure on Phospholipid Vesicle Formation. Soft Matter 2017, 13 (7), 1364-1370.

21. López-Barrón, C. R.; Li, D.; DeRita, L.; Basavaraj, M. G.; Wagner, N. J., Spontaneous Thermoreversible Formation of Cationic Vesicles in a Protic Ionic Liquid. J. Am. Chem. Soc. 2012, 134 (51), 20728-32.

22. Abbott, A. P.; Capper, G.; Davies, D. L.; Rasheed, R. K.; Tambyrajah, V., Novel Solvent Properties of Choline Chloride/Urea Mixtures. Chem. Commun. 2003, (1), 70-71.

23. Abbott, A. P.; Boothby, D.; Capper, G.; Davies, D. L.; Rasheed, R. K., Deep Eutectic Solvents Formed between Choline Chloride and Carboxylic Acids: Versatile Alternatives to Ionic Liquids. $J$. Am. Chem. Soc. 2004, 126 (29), 9142-9147.

24. Abbott, A. P.; Harris, R. C.; Ryder, K. S.; D'Agostino, C.; Gladden, L. F.; Mantle, M. D., Glycerol Eutectics as Sustainable Solvent Systems. Green Chemistry 2011, 13 (1), 82-90.

25. Smith, E. L.; Abbott, A. P.; Ryder, K. S., Deep Eutectic Solvents (Dess) and Their Applications. Chem. Rev. 2014, 114 (21), 11060-11082.

26. Zhang, Q.; De Oliveira Vigier, K.; Royer, S.; Jerome, F., Deep Eutectic Solvents: Syntheses, Properties and Applications. Chem. Soc. Rev. 2012, 41 (21), 7108-7146. 
27. Bryant, S. J.; Atkin, R.; Warr, G. G., Spontaneous Vesicle Formation in a Deep Eutectic Solvent. Soft Matter 2016, 12 (6), 1645-1648.

28. Sanchez-Fernandez, A.; Edler, K. J.; Arnold, T.; Heenan, R. K.; Porcar, L.; Terrill, N. J.; Terry, A. E.; Jackson, A. J., Micelle Structure in a Deep Eutectic Solvent: A Small-Angle Scattering Study. Phys. Chem. Chem. Phys. 2016, 18 (20), 14063-14073.

29. Arnold, T.; Jackson, A. J.; Sanchez-Fernandez, A.; Magnone, D.; Terry, A. E.; Edler, K. J., Surfactant Behavior of Sodium Dodecylsulfate in Deep Eutectic Solvent Choline Chloride/Urea. Langmuir 2015, 31 (47), 12894-12902.

30. Pal, M.; Singh, R. K.; Pandey, S., Evidence of Self-Aggregation of Cationic Surfactants in a Choline Chloride+Glycerol Deep Eutectic Solvent. ChemPhysChem 2015, 16 (12), 2538-2542.

31. Abbott, A. P.; Capper, G.; Gray, S., Design of Improved Deep Eutectic Solvents Using Hole Theory. ChemPhysChem 2006, 7 (4), 803-806.

32. Chen, Z.; Ludwig, M.; Warr, G. G.; Atkin, R., Effect of Cation Alkyl Chain Length on Surface Forces and Physical Properties in Deep Eutectic Solvents. J. Colloid Interface Sci. 2017, 494, 373379.

33. Chen, Z. F.; Greaves, T. L.; Warr, G. G.; Atkin, R., Mixing Cations with Different Alkyl Chain Lengths Markedly Depresses the Melting Point in Deep Eutectic Solvents Formed from Alkylammonium Bromide Salts and Urea. Chem. Commun. (Cambridge, U. K.) 2017, 53 (15), 23752377.

34. Hayes, R.; Imberti, S.; Warr, G. G.; Atkin, R., Effect of Cation Alkyl Chain Length and Anion Type on Protic Ionic Liquid Nanostructure. J. Phys. Chem. C 2014, 118, 13998-13998.

35. Hayes, R.; Imberti, S.; Warr, G. G.; Atkin, R., Amphiphilicity Determines Nanostructure in Protic Ionic Liquids. Phys. Chem. Chem. Phys. 2011, 13 (8), 3237-3247.

36. Greaves, T. L.; Kennedy, D. F.; Kirby, N.; Drummond, C. J., Nanostructure Changes in Protic Ionic Liquids (Pils) through Adding Solutes and Mixing Pils. Phys. Chem. Chem. Phys. 2011, 13 (30), 13501-13509

37. Hayes, R.; Imberti, S.; Warr, G. G.; Atkin, R., How Water Dissolves in Protic Ionic Liquids. Angew. Chem., Int. Ed. 2012, 51 (30), 7468-7471.

38. Murphy, T.; Hayes, R.; Imberti, S.; Warr, G. G.; Atkin, R., Nanostructure of an Ionic LiquidGlycerol Mixture. Phys. Chem. Chem. Phys. 2014, 16 (26), 13182-13190.

39. Jiang, H. J.; FitzGerald, P. A.; Dolan, A.; Atkin, R.; Warr, G. G., Amphiphilic Self-Assembly of Alkanols in Protic Ionic Liquids. J. Phys. Chem. B 2014, 118 (33), 9983-90.

40. Murphy, T.; Callear, S. K.; Warr, G. G.; Atkin, R., Dissolved Chloride Markedly Changes the Nanostructure of the Protic Ionic Liquids Propylammonium and Ethanolammonium Nitrate. Phys. Chem. Chem. Phys. 2016, 18 (26), 17169-17182.

41. Evans, D. F.; Kaler, E. W.; Benton, W. J., Liquid Crystals in a Fused Salt: B, ГDistearoylphosphatidylcholine in $N$-Ethylammonium Nitrate. J. Phys. Chem. 1983, 87 (4), 533-535.

42. Choi, Y. H.; van Spronsen, J.; Dai, Y.; Verberne, M.; Hollmann, F.; Arends, I. W. C. E.; Witkamp, G.-J.; Verpoorte, R., Are Natural Deep Eutectic Solvents the Missing Link in Understanding Cellular Metabolism and Physiology? Plant Physiology 2011, 156 (4), 1701-1705.

43. Farrant, J. M.; Cooper, K.; Hilgart, A.; Abdalla, K. O.; Bentley, J.; Thomson, J. A.; Dace, H. J. W.; Peton, N.; Mundree, S. G.; Rafudeen, M. S., A Molecular Physiological Review of Vegetative Desiccation Tolerance in the Resurrection Plant Xerophyta Viscosa (Baker). Planta 2015, 242 (2), 407-426.

44. Laughlin, R. G., The Role of Swelling Methods in Surfactant Phase Science: Past, Present, and Future. Adv. Colloid Interface Sci. 1992, 41 (0), 57-79.

45. Rosevear, F. B., The Microscopy of the Liquid Crystalline Neat and Middle Phases of Soaps and Synthetic Detergents. Journal of the American Oil Chemists' Society 1954, 31 (12), 628-639.

46. Shanks, R. A.; Staszczyk, D., Thermal and Optical Characterization of Polymer-Dispersed Liquid Crystals. International Journal of Polymer Science 2012, 2012, 13. 
47. Shipley, G. G.; Avecilla, L. S.; Small, D. M., Phase Behavior and Structure of Aqueous Dispersions of Sphingomyelin. J. Lipid Res. 1974, 15 (2), 124-131.

48. Laughlin, R. G., The Aqueous Phase Behaviour of Surfactants. Academic Press: San Diego, CA 92101, 1994; p 558.

49. Swamy, M. J.; Marsh, D., Thermodynamics of Interdigitated Phases of Phosphatidylcholine in Glycerol. Biophysical Journal 1995, 69 (4), 1402-1408.

50. Lewis, B. A.; Engelman, D. M., Lipid Bilayer Thickness Varies Linearly with Acyl ChainLength in Fluid Phosphatidylcholine Vesicles. J. Mol. Biol. 1983, 166 (2), 211-217.

51. Kucerka, N.; Liu, Y. F.; Chu, N. J.; Petrache, H. I.; Tristram-Nagle, S. T.; Nagle, J. F., Structure of Fully Hydrated Fluid Phase Dmpc and Dlpc Lipid Bilayers Using X-Ray Scattering from Oriented Multilamellar Arrays and from Unilamellar Vesicles. Biophys. J. 2005, 88 (4), 2626-2637.

52. Tristram-Nagle, S.; Liu, Y. F.; Legleiter, J.; Nagle, J. F., Structure of Gel Phase Dmpc Determined by X-Ray Diffraction. Biophys. J. 2002, 83 (6), 3324-3335.

53. Chen, X.; Hua, W.; Huang, Z.; Allen, H. C., Interfacial Water Structure Associated with Phospholipid Membranes Studied by Phase-Sensitive Vibrational Sum Frequency Generation Spectroscopy. J. Am. Chem. Soc. 2010, 132 (32), 11336-11342.

54. Ohto, T.; Backus, E. H. G.; Hsieh, C.-S.; Sulpizi, M.; Bonn, M.; Nagata, Y., Lipid Carbonyl Groups Terminate the Hydrogen Bond Network of Membrane-Bound Water. The Journal of Physical Chemistry Letters 2015, 6 (22), 4499-4503.

55. Morgan, J.; Napper, D.; Warr, G.; Nicol, S., Measurement of the Selective Adsorption of Ions at Air/Surfactant Solution Interfaces. Langmuir 1994, 1 (16), 797-801.

56. Thalody, B.; Warr, G. G., The Selective Binding of Carboxylate Ions at Cationic Surfactant Solution/Air Interfaces. J. Colloid Interface Sci. 1997, 188 (2), 305-312.

57. Aroti, A.; Leontidis, E.; Dubois, M.; Zemb, T., Effects of Monovalent Anions of the Hofmeister Series on Dppc Lipid Bilayers Part I: Swelling and in-Plane Equations of State. Biophysical Journal 2007, 93 (5), 1580-1590.

58. Aroti, A.; Leontidis, E.; Dubois, M.; Zemb, T.; Brezesinski, G., Monolayers, Bilayers and Micelles of Zwitterionic Lipids as Model Systems for the Study of Specific Anion Effects. Colloids and Surfaces A: Physicochemical and Engineering Aspects 2007, 303 (1-2), 144-158.

59. Clarke, R. J.; Lüpfert, C., Influence of Anions and Cations on the Dipole Potential of Phosphatidylcholine Vesicles: A Basis for the Hofmeister Effect. Biophysical Journal 1999, 76 (5), 2614-2624.

60. Greaves, T. L.; Drummond, C. J., Solvent Nanostructure, the Solvophobic Effect and Amphiphile Self-Assembly in Ionic Liquids. Chem.Soc.Rev. 2013, 42, 1096-1120.

61. Hayes, R.; Imberti, S.; Warr, G. G.; Atkin, R., Pronounced Sponge-Like Nanostructure in Propylammonium Nitrate. Phys. Chem. Chem. Phys. 2011, 13 (30), 13544-13551.

62. Umebayashi, Y.; Chung, W.; Mitsugi, T.; Fukuda, S.; Takeuchi, M.; Fujii, K.; Takamuku, T.; Kanzaki, R.; Ishiguro, S., Liquid Structure and the Ion-Ion Interactions of Ethylammonium Nitrate Ionic Liquid Studied by Large Angle X-Ray Scattering and Molecular Dynamics Simulations. $J$. Comput. Chem. Jpn. 2008, 7 (4), 125-134.

63. Greaves, T. L.; Kennedy, D. F.; Mudie, S. T.; Drummond, C. J., Diversity Observed in the Nanostructure of Protic Ionic Liquids. J. Phys. Chem. B 2010, 114 (31), 10022-10031.

64. Atkin, R.; Warr, G. G., The Smallest Amphiphiles: Nanostructure in Protic Room-Temperature Ionic Liquids with Short Alkyl Groups. J. Phys. Chem. B 2008, 112 (14), 4164-4166.

65. Zana, R.; Yiv, S.; Strazielle, C.; Lianos, P., Effect of Alcohol on the Properties of Micellar Systems. J. Colloid Interface Sci. 1981, 80 (1), 208-223.

66. Lianos, P.; Zana, R., Fluorescence Probing Study of the Effect of Medium Chain-Length Alcohols on the Properties of Tetradecyltrimethylammonium Bromide Aqueous Micelles. Chem. Phys. Lett. 1980, 76 (1), 62-67. 
67. Rowe, E. S.; Zhang, F.; Leung, T. W.; Parr, J. S.; Guy, P. T., Thermodynamics of Membrane Partitioning for a Series of N-Alcohols Determined by Titration Calorimetry: Role of Hydrophobic Effects. Biochemistry 1998, 37 (8), 2430-2440.

68. Westh, P.; Trandum, C., Partitioning of Small Alcohols into Dimyristoyl Phosphatidylcholine (Dmpc) Membranes: Volumetric Properties. The Journal of Physical Chemistry B 2000, 104 (47), 11334-11341.

69. Greaves, T. L.; Drummond, C. J., Solvent Nanostructure, the Solvophobic Effect and Amphiphile Self-Assembly in Ionic Liquids. Chem. Soc. Rev. 2013, 42 (3), 1096-1120.

70. Friberg, S., Organized Solutions: Surfactants in Science and Technology. Taylor \& Francis: 1992.

71. Hamel, A.; Sacco, M.; Mnasri, N.; Lamaty, F.; Martinez, J.; De Angelis, F.; Colacino, E.; Charnay, C., Micelles into Glycerol Solvent: Overcoming Side Reactions of Glycerol. ACS Sustainable Chemistry \& Engineering 2014, 2 (6), 1353-1358.

72. Nagarajan, R.; Wang, C.-C., Solution Behavior of Surfactants in Ethylene Glycol: Probing the Existence of a Cmc and of Micellar Aggregates. J. Colloid Interface Sci. 1996, 178 (2), 471-482.

73. Palepu, R.; Gharibi, H.; Bloor, D. M.; Wyn-Jones, E., Electrochemical Studies Associated with the Micellization of Cationic Surfactants in Aqueous Mixtures of Ethylene Glycol and Glycerol. Langmuir 1993, 9 (1), 110-112.

74. Carnero Ruiz, C., Thermodynamics of Micellization of Tetradecyltrimethylammonium Bromide in Ethylene Glycol-Water Binary Mixtures. Colloid Polym Sci 1999, 277 (7), 701-707.

75. Israelachvili, J. N., Mitchell, D.J., Ninham, B.W., Theory of Self-Assembly of Hydrocarbon Amphiphiles into Micelles and Bilayers. J. Chem. Soc. Faraday Trans. 2 1976, 72, 1526-1568.

76. Hayes, R.; Imberti, S.; Warr, G. G.; Atkin, R., The Nature of Hydrogen Bonding in Protic Ionic Liquids. Angewandte Chemie-International Edition 2013, 52 (17), 4623-4627.

77. Sharma, S. C.; Atkin, R.; Warr, G. G., The Effect of Ionic Liquid Hydrophobicity and Solvent Miscibility on Pluronic Amphiphile Self-Assembly. J. Phys. Chem. B 2013, 117 (46), 14568-14575.

78. Pal, M.; Rai, R.; Yadav, A.; Khanna, R.; Baker, G. A.; Pandey, S., Self-Aggregation of Sodium Dodecyl Sulfate within (Choline Chloride + Urea) Deep Eutectic Solvent. Langmuir 2014, 30 (44), 13191-13198.

79. Thomas, D. N.; Dieckmann, G. S., Antarctic Sea Ice--a Habitat for Extremophiles. Science 2002, 295 (5555), 641-644.

80. Ojha, L.; Wilhelm, M. B.; Murchie, S. L.; McEwen, A. S.; Wray, J. J.; Hanley, J.; Masse, M.; Chojnacki, M., Spectral Evidence for Hydrated Salts in Recurring Slope Lineae on Mars. Nature Geosci 2015, 8 (11), 829-832.

81. Gillon, M.; Triaud, A. H. M. J.; Demory, B.-O.; Jehin, E.; Agol, E.; Deck, K. M.; Lederer, S. M.; de Wit, J.; Burdanov, A.; Ingalls, J. G.; Bolmont, E.; Leconte, J.; Raymond, S. N.; Selsis, F.; Turbet, M.; Barkaoui, K.; Burgasser, A.; Burleigh, M. R.; Carey, S. J.; Chaushev, A.; Copperwheat, C. M.; Delrez, L.; Fernandes, C. S.; Holdsworth, D. L.; Kotze, E. J.; Van Grootel, V.; Almleaky, Y.; Benkhaldoun, Z.; Magain, P.; Queloz, D., Seven Temperate Terrestrial Planets around the Nearby Ultracool Dwarf Star Trappist-1. Nature 2017, 542 (7642), 456-460. 